\title{
Intervenir pour le développement et l'innovation : l'organisation communautaire dans les réseaux public et associatif
}

\author{
Yvan Comeau, Université Laval \\ Martine Duperré, Université Laval \\ Yves Hurtubise, Université Laval \\ Clément Mercier, Université de Sherbrooke \\ Daniel Turcotte, Université Laval
}

\section{Introduction}

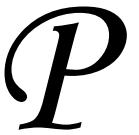

our le succès d'une démarche de développement et d'innovation, les ressources d'accompagnement apportent une contribution essentielle ${ }^{1}$. Plus particulièrement, la présence de personnes spécialisées prodiguant conseil, information, éducation, mise en réseau et encouragement s'avère nécessaire pour la réussite des initiatives collectives $^{2}$. Sur ce type d'intervention, il existe une riche tradition académique, théorique et empirique ${ }^{3}$, le plus souvent associée au travail social et que l'on désigne sous le vocable « organisation communautaire». Bien des professionnels du développement pratiquent l'organisation communautaire sans pourtant s'en réclamer. On peut désigner l'organisation communautaire

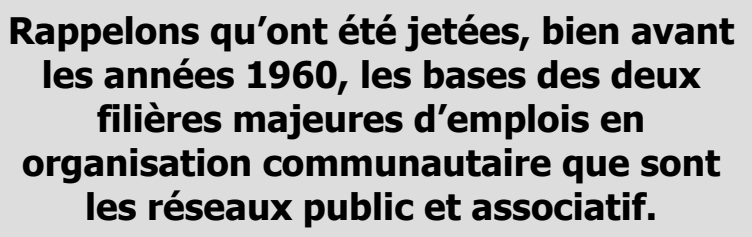

On retrouve des organisateurs communautaires (OC) dans différents établissements publics (en particulier les Centres de services sociaux - CSS) et dans divers organismes. Ces personnes appuient la création et l'émergence de diverses initiatives collectives, d'organismes de développement, d'entreprises d'économie sociale et d'associations qui constituent autant d'innovations pour le développement des collectivités. Afin de connaitre les caractéristiques des OC du Québec et les principaux paramètres de leur pratique, une recherche a été entreprise ${ }^{5}$. Dans cet article, l'équipe présente certains résultats de la recherche qui mettent en évidence l'influence de l'établissement-employeur sur

l'organisation communautaire puisque celuici représente une des principales sources d'influence qui s'exercent sur l'organisation communautaire, avec comme étant une intervention salariée et orientée vers la solidarité et la participation sociale au moyen de pratiques démocratiques avec un groupe de personnes, une ou plusieurs associations, ou une collectivité de type territorial, identitaire ou d'intérêt. Cette intervention est pratiquée selon un processus par lequel le groupe, l'association ou la collectivité identifie des problèmes, mobilise des ressources et développe une action collective pour y répondre ${ }^{4}$.

Par cette définition, on comprend que l'organisation communautaire favorise une orientation progressiste du développement et appuie les innovations sociales dans la mesure où ces pratiques inédites dans un milieu donné peuvent répondre à des besoins sociaux et permettre leur appropriation collective.
l'État et les communautés. Ainsi croyons-nous contribuer à la connaissance des dynamiques de développement en examinant une catégorie de professionnels qui accompagnent les acteurs préoccupés par la revitalisation de leur communauté.

Rappelons qu'ont été jetées, bien avant les années 1960, les bases des deux filières majeures d'emplois en organisation communautaire que sont les réseaux public et associatif. Avant 1960, l'organisation communautaire était l'œuvre de pionniers (religieux, agronomes, syndicalistes et bénévoles), sans statut professionnel associé à une telle fonction, mais qui néanmoins initiaient et supportaient des actions collectives répondant à des besoins et pouvant entraîner des changements sociaux. Puis, au cours de la période 
1960-1975, l'organisation communautaire représentait une des sources d'inspiration pour procéder au rattrapage du Québec et à sa modernisation, et constituait elle-même une véritable innovation dans le contexte de la croissance économique d'après-guerre et de la mise en place des politiques sociales. Une des étapes importantes de la diffusion de l'organisation communautaire coïncide avec la Loi sur la santé et les services sociaux, adoptée en 1970 par le gouvernement du Québec, qui instituait ainsi les Centres locaux de services communautaires (CLSC). Dès le début de leur fonctionnement en 1974, ceux-ci accordaient une place particulière aux OC; d'ailleurs, dans bien des cas, les OC faisaient partie du groupe fondateur de CLSC. De mieux en mieux connue au milieu des années 1970, l'organisation communautaire commençait à être considérée comme un métier : en 1976, la convention collective des employés de l'État a défini les tâches d'un OC et il s'est constitué des associations pour regrouper ses praticiens ${ }^{6}$. À mesure que le réseau associatif se structurait, souvent d'ailleurs avec l'appui d'OC du réseau public, les ressources financières que mobilisaient un nombre croissant d'associations et d'organismes parapublics favorisaient l'embauche de salariés pratiquant l'organisation communautaire ${ }^{7}$.

Afin de circonscrire les modalités de la contribution de l'organisation communautaire au développement local, cet article présente d'abord les principaux aspects méthodologiques de l'étude réalisée. Par la suite, le texte fait place à quelques résultats de la recherche qui concernent d'abord les caractéristiques des établissements-employeurs et leur influence sur la pratique de l'organisation communautaire. Puis sont examinées les caractéristiques sociales et profession- nelles des personnes qui pratiquent l'organisation communautaire ainsi que les pratiques qu'elles déploient afin de contribuer, à leur manière, au développement social et économique des communautés et à la diffusion des innovations sociales.

\section{Les paramètres de la recherche}

Pour mieux délimiter la pratique de l'organisation communautaire dans les établissements publics et les associations, l'équipe de recherche a développé un questionnaire portant sur la situation professionnelle des OC telle qu'elle se présentait en décembre 2003, soit avant l'adoption des lois québécoises ayant pour effet de modifier le réseau de la santé et des services sociaux. Ce questionnaire a été acheminé à deux catégories de personnes : 1) tous les $\mathrm{OC}$ à l'embauche des CLSC du Québec et 2) tous les OC des autres établissements et organismes ${ }^{8}$ situés dans les régions de Québec, de 1'Estrie et de Chaudière-Appalaches ${ }^{9}$. Avec ce dernier groupe, il a d'abord fallu repérer les personnes qui pratiquent l'organisation communautaire. Pour y arriver, les membres de l'équipe ont constitué une liste d'organismes susceptibles d'avoir au moins un $\mathrm{OC}$ à leur emploi. Puis, lors d'un contact téléphonique et à l'aide d'une définition de l'organisation communautaire ${ }^{10}$, ont été identifiées les personnes affirmant la pratiquer dans leur emploi. Cette procédure a permis de repérer 255 OC dans divers organismes de la région de Québec, 110 en Estrie et 73 dans Chaudière-Appalaches. Au total, 48,8 \% des personnes sollicitées ont rempli le questionnaire qui leur a été envoyé par la poste. Le tableau 1 permet de comparer le nombre de répondants en regard de l'ensemble des OC repérés, selon le type d'organismeemployeur.

\section{Tableau 1 - Comparaison entre l'échantillon et l'ensemble des répondants selon le type d'organisme}

\begin{tabular}{|c|c|c|c|c|}
\hline & \multicolumn{2}{|c|}{ Échantillon } & \multicolumn{2}{|c|}{ Population } \\
\hline & $\mathrm{N}$ & $\%$ & $\mathrm{~N}$ & $\%$ \\
\hline Établissements publics & 211 & 50,8 & 411 & 48,4 \\
\hline Autres organismes ${ }^{1}$ & 204 & 49,2 & 438 & 51,6 \\
\hline Total & 415 & 100,0 & 849 & 100,0 \\
\hline
\end{tabular}

Khi deux $=0,692 ; 1$ d. 1.; $p>0,05$. Différence non significative.

Note : Parmi ces organismes, on compte 189 organismes communautaires et populaires. 
L'échantillon ne présente pas de différence significative avec la population à l'étude non seulement sur la base du type d'organisme-employeur (tableau 1), mais également selon le sexe des répondants $\left(\mathrm{X}^{2}=\right.$ $0,007 ; \mathrm{p}>, 05)$. Étant donné que sont disponibles les réponses de 211 OC du réseau public et de 189 OC du réseau associatif, il devient alors possible d'effectuer des comparaisons. Les différences évoquées dans ce texte se sont avérées significatives puisque les tests statistiques non paramétrique (le khi deux de Pearson) et paramétrique (ANOVA) atteignaient un seuil de signification de $95,0 \%$ (les différences observées sont significatives 19 fois sur 20).

\section{L'identité suscitée par l'expression « organisation communautaire » est plus forte dans le réseau public que dans le réseau associatif.}

\section{Des organisations de vocation et de taille différentes}

D'après les informations recueillies auprès des répondants, les deux tiers œuvrent dans des organismes $(63,6 \%)$ qui rejoignent une population constituée à la fois de personnes à faible et moyen revenu. Cette proportion est beaucoup plus forte chez les répondants des établissements publics que chez ceux des associations ( $70,4 \%$ des premiers et $55,3 \%$ des secondes). Dans ce dernier groupe, plus de quatre personnes sur dix (41,2\% des répondants) indiquent que leur organisme rejoint principalement une population «très majoritairement à faibles revenus », comparativement à moins de un sur cinq $(18,4 \%)$ dans le réseau public.

D'après les réponses obtenues, en 2003, le nombre moyen d'employés dans les établissements publics au sein desquels auvrent les OC est de 362,9 personnes alors que dans les associations, la moyenne se situe à 16,8. Les OC du réseau public sont intégrés dans des équipes beaucoup plus imposantes; dans ce type d'organisme, le nombre de personnes sous la responsabilité du supérieur immédiat est presque huit fois supérieur (moyenne de 50,3 personnes) à celui observé dans les associations (6,6 personnes).

Les OC du réseau associatif disent plus souvent ne pas avoir de supérieur salarié $(44,7 \%$ de ces OC comparativement à $2,4 \%$ des OC du réseau public). Parmi les raisons qui peuvent expliquer l'absence de supérieur immédiat dans le réseau associatif, il y a le fait que la responsabilité de gestion du personnel soit assumée par le conseil d'administration (lui-même élu par l'assemblée des membres) et le fait que les OC occupent eux-mêmes des postes d'encadrement à titre de « coordonnateur» ou « directeur général ». D’ailleurs, dans le réseau associatif, 30,0\% des répondants se définissent comme des coordonnateurs, comparativement à aucun dans le réseau public.

\section{Des titres d'emploi fort variés dans le réseau associatif}

Bien que le titre «organisateur communautaire» (OC) désigne l'emploi de près de la moitié des répondants (48,3\% des répondants), il existe tout de même un certain éclatement dans la manière de nommer ce métier, surtout dans le réseau associatif. De fait, ce titre prévaut largement dans le réseau public $(92,4 \%$ des répondants), alors qu'on retrouve une diversité d'appellations dans le réseau associatif (coordonnateur, chargé de projet, intervenant, conseiller, agent de développement, de milieu, d'information, etc.). On peut dès lors supposer que l'identité suscitée par l'expression " organisation communautaire » est plus forte dans le réseau public que dans le réseau associatif.

\section{Des caractéristiques sociales des OC propres à chaque réseau}

Nos données traduisent des profils d'OC différents selon le type de réseau. Premièrement, même si les femmes constituent la majorité des OC $(59,7 \%$ des répondants), les hommes se retrouvent, au moment de l'enquête, en proportion plus importante dans les établissements publics $(65,5 \%)$. Deuxièmement, on observe un écart important quant à l'âge : plus de la moitié des répondants œuvrant dans les associations ont moins de 41 ans $(55,1 \%)$, alors que ce pourcentage se situe à moins de un sur cinq dans le réseau public $(18,9 \%)$. Troisièmement, les OC du réseau public occupent leur emploi depuis beaucoup plus longtemps; ils ont en moyenne 13 années d'ancienneté comparativement à 4,5 années pour les $\mathrm{OC}$ du réseau associatif. Cette différence nous apparaît traduire un taux de roulement du personnel plus important dans le réseau associatif. Quatrièmement, les $\mathrm{OC}$ $\mathrm{du}$ réseau public sont davantage scolarisés que leurs homologues du réseau associatif. En effet, presque neuf répondants du réseau public sur $10(87,4 \%)$ possèdent au moins un baccalauréat, alors que six répon- 
dants du réseau communautaire sur $10(61,3 \%)$ en ont un. On comprend cette situation dans la mesure où plusieurs incitatifs à la scolarisation existent dans le réseau public soit à l'embauche, soit pour se positionner dans les échelles salariales. Cinquièmement, la discipline du travail social s'avère importante puisque plus de quatre répondants sur dix ont une formation dans ce domaine, soit $52,6 \%$ des OC dans les établissements publics et $30,1 \%$ dans le réseau associatif. Les autres disciplines sont principalement: psychologie, sociologie, sciences de l'éducation, théologie, animation, sciences de la santé, gestion et administration. Pour expliquer cette différence entre les types d'établissement, on peut faire l'hypothèse que le réseau de la santé et des services sociaux a eu tendance à privilégier l'embauche de travailleurs sociaux pour pratiquer l'organisation communautaire en raison des exigences des conventions collectives.

\section{Les conditions de travail}

Chez les répondants, plus de huit sur dix $(85,6 \%)$ indiquent qu'ils occupent un poste permanent ou régulier. Cette proportion varie entre le type de réseau; elle se situe à $90,4 \%$ dans le réseau public et à $80,6 \%$ dans les associations $\left(\mathrm{X}^{2}=8,043 ; \mathrm{p}=, 005\right)$. Par ailleurs, dans les deux réseaux, la proportion des emplois à temps complet ne diffère pas; elle se situe à $85,7 \%$ pour l'ensemble des répondants. Les conditions salariales présentent cependant un profil très différent. Ainsi, le salaire annuel mentionné par les OC du réseau public se situe en moyenne à $51161 \$$, comparativement à $25400 \$$, affirment leurs homologues du réseau associatif.

Il n'est donc pas étonnant que plus de six répondants sur dix $(60,5 \%)$ dans le réseau public soient « fortement » ou «très fortement» en accord avec l'énoncé : «Mes conditions de travail sont excellentes », comparativement au quart $(26,7 \%)$ dans les associations. Cependant, en ce qui a trait à la satisfaction générale au travail, il n'y a pas de différence entre les deux groupes de répondants. Toutefois, on décèle une volonté de mobilité plus grande chez les répondants qui proviennent du réseau associatif. En ce qui a trait à l'autonomie au travail et à l'impact de ce dernier sur la santé, les répondants formulent majoritairement une appréciation positive. L'opinion sur ces aspects ne diffère pas selon le réseau (alors qu'on pourrait croire que l'autonomie est davantage appréciée des salariés en milieu associatif) ni selon l'âge des répondants. On observe toutefois une différence selon le sexe : les répondants de sexe féminin ont davantage indiqué «qu'on leur en demande trop, qu'elles sont débordées au travail » $(15,7 \%$ des femmes sont de cet avis comparativement à 7,3\% des hommes).

On peut expliquer en partie les écarts salariaux entre les réseaux par l'ancienneté acquise par les OC du réseau public et par leur formation supérieure. Mais il faut sans doute insister également sur la syndicalisation du réseau public et sur les acquis des luttes des employés de l'État pour des conventions collectives offrant les conditions de travail que l'on connait.

\section{Le ressourcement privilégié dans le réseau associatif}

Le perfectionnement repose d'abord sur des «sessions de formation », c'est-à-dire des activités circonscrites dans le temps, ne conduisant pas nécessairement à un diplôme (bien que des répondants mentionnent des activités créditées par un établissement d'enseignement) et visant à améliorer la pratique de l'organisation communautaire, comme le stipulait la question. Ce type de formation s'avère beaucoup plus fréquent que la formation continue conduisant à un diplôme : 68,2 \% des répondants affirment avoir suivi de telles sessions (comparativement à environ $15 \%$ des répondants qui poursuivent des études). Plus souvent que les OC du réseau public, les OC du réseau associatif assistent à ces « sessions de formation ».

\section{La pratique de l'organisation communautaire}

La pratique de l'organisation réfère à des valeurs, à des courants d'idées, à des conceptions de l'organisation communautaire et à des tâches. En ce qui concerne les valeurs les plus souvent évoquées pour appuyer sa pratique, on retrouve, dans l'ordre : la solidarité, l'autonomie, la justice, l'égalité et l'authenticité. Il existe des différences significatives entre les OC des réseaux public et associatif. Les premiers privilégient les valeurs de solidarité et de justice, alors que les seconds optent davantage pour les valeurs d'égalité et d'authenticité.

À l'égard des divers courants d'idées proposés dans le questionnaire, un premier groupe se démarque. Le plus gros, ce groupe se distingue des deux autres par 
son adhésion plus faible à une vision de l'organisation communautaire axée sur la «politisation et l'éducation politique »; il privilégie plutôt la «concertation du milieu » et la « prise en charge du milieu ». Adhérant davantage au courant de la " conscientisation", le deuxième groupe est fortement féminin, plutôt jeune, se retrouve principalement à l'extérieur du réseau public et compte peu d'ancienneté dans son milieu de travail. Un troisième groupe, composé majoritairement d'hommes, s'inspire de l' " alternative » et des «mouvements sociaux ».

De manière générale, la conception la plus répandue de l'organisation communautaire est celle de l'" organisation des services » $(35,2 \%$ des répondants $)$, c'est-à-dire une intervention qui appuie ou initie la mise sur pied de services axés sur l'amélioration des conditions de vie. Suit une conception de l'organisation communautaire qui combine différents types d'intervention, soit l'animation de groupes, l'organisation de services et d'entreprises communautaire, le soutien aux actions des groupes populaires et l'éducation politique (34,0\% des répondants). On retrouve comme troisième conception une vision de l'organisation communautaire axée sur l' " animation de groupe »; ce choix est exprimé par près d'une personne sur quatre (24,6\% des choix). Il est intéressant de noter que les interventions de type «action sociale » ( « participation et pression » $[14,7 \%]$ et « politisation et éducation » $[(8,9 \%])$ sont privilégiées par moins de $15 \%$ des répondants.

Des différences importantes existent quant à la conception de l'organisation communautaire. Les OC du réseau public insistent davantage sur une conception de l'organisation communautaire incluant tous les types d'intervention; en outre, ils se réfèrent plus souvent à l'" organisation d'entreprises communautaires ». Quant aux OC du réseau associatif, les conceptions de l'organisation communautaire en tant qu' « organisation de services», d' «animation de groupes » et de " politisation - éducation politique » les rejoignent davantage.

L'analyse révèle que des différences significatives existent entre les réseaux quant aux domaines d'intervention. Les OC du réseau associatif œuvrent plus souvent que leurs homologues du réseau public dans des projets «socioculturels» et plus particulièrement en «éducation populaire». En ce qui concerne les projets de type «sociosanitaire », les OC du réseau public s'attardent davantage au "maintien à domicile ». Pour ce qui est des projets de nature «socioéconomique », les OC de l'associatif développent proportionnellement moins de projets de « développement local » que ceux du réseau public, bien que ce type de projets demeure le plus important parmi les projets «socio-économiques» menés par ces employés du réseau associatif.

Il s'avère que l'organisation communautaire ne vise pas les mêmes groupes socio-économiques selon qu'elle est pratiquée dans les établissements publics ou dans les associations. D'après les données recueillies, les OC du réseau public se préoccupent davantage que leurs collègues de l'associatif, de la «population en général», des "groupes communautaires » et des «parents ». Les OC du réseau associatif rejoignent plus souvent que les précédents, les « jeunes (12 à 30 ans) » et les "femmes (condition féminine) ».

Sur une base comparative, les OC du réseau public assument plus souvent des fonctions autour des réunions et assemblées (animation et organisation), de la création d'organismes («aide à l'émergence de groupes » et « mise en place de coalitions ») et du conseil (« formation et conseil », « aide au développement de stratégies » et « appui à une instance décisionnelle »). Dans le réseau associatif, on demande davantage aux OC d'assumer des fonctions entourant l'organisation d'actions et la gestion de ressources financières et humaines (salariées et bénévoles).

\section{La conception la plus répandue de I'organisation communautaire est celle de I'« organisation des services ».}

L'animation de «tables de concertation » est nettement le fait des OC du réseau public; $91,9 \%$ indiquent faire cette tâche comparativement à $42,6 \%$ dans les associations. En outre, ces personnes sont plus souvent impliquées dans les équipes inter ou multidisciplinaires, dans des associations, des groupes d'action, des coalitions et des conseils consultatifs. Chez les OC du réseau associatif, les comités représentent le type de groupe le plus souvent animés (70,6\% des répondants). Les conseils d'administration, les groupes de supervision ou de formation, les comités d'étude de cas et les diverses instances de 
direction font partie des groupes de tâches les plus fréquemment animés.

La proportion du temps de travail qui est consacrée à l'organisation communautaire est nettement plus importante dans les CLSC que dans les organismes communautaires $(86,1 \%$ pour le premier et $68,6 \%$ pour le deuxième). Cela tend à confirmer l'hypothèse que dans le milieu associatif, les intervenants sont appelés à exercer plusieurs tâches, dont celles liées à la gestion.

\section{Le rattachement organisationnel exerce une influence considérable sur l'organisation communautaire.}

\section{Conclusion}

Cet article a mis en évidence quelques caractéristiques de l'organisation communautaire dans les réseaux public et associatif ainsi que certains effets du contexte de travail sur la pratique. Il ressort des résultats de la recherche que l'établissement-employeur marque d'abord l'organisation communautaire par certaines règles externes comme, par exemple, le fait de la syndicalisation dans le réseau public. Puis, la structure politique interne de l'établissement détermine la forme hiérarchique dans laquelle doivent s'insérer les OC. Cette structure politique n'est pas étrangère au statut juridique de l'établissement de même qu'à l'ampleur et à la provenance de ses ressources. Par ailleurs, la mission que se donne l'établissement, avec plus ou moins de marge de manœuvre lorsqu'on tient compte des orientations prises par les ministères et les bailleurs de fonds, agit sur la pratique de l'organisation communautaire. Ainsi, les établissements publics affichent plus souvent une mission assez large couvrant une gamme étendue d'activités sur des territoires parfois vastes ou encore populeux; on comprend que les OC qui y œuvrent amalgament différentes conceptions de l'organisation communautaire pour mener une diversité de mandats. Du côté des associations, les missions sont plus spécifiques et favorisent, du même coup, des pratiques plus circonscrites. Par ailleurs, le profil des tâches assumées par les OC du réseau public est influencé par le fait qu'ils interviennent plus souvent avec des organismes et des groupes, alors que les $\mathrm{OC}$ du réseau associatif œuvrent dans un organisme qui fait appel à eux pour la gestion et pour l'organisation communautaire qui n'occupe qu'une partie de leur temps.

Les résultats de la recherche montrent ainsi que le rapport des intervenants professionnels avec les initiatives de développement et les innovations sociales varie considérablement selon l'organisme-employeur. Il est donc confirmé que le rattachement organisationnel exerce une influence considérable sur l'organisation communautaire et que la détermination d'une orientation pour sa pratique professionnelle débute véritablement dans le choix de ce rattachement.

\section{Notes et références}

1 Klein, J.-L. (1992). « Le partenariat : vers une planification flexible du développement local », Revue canadienne des sciences régionales, vol. XV, $\mathrm{n}^{\mathrm{o}} 3$, p. 491-505; Favreau, L. et B. Lévesque (1996). Développement économique communautaire. Économie sociale et intervention, Sainte-Foy, Presses de l'Université du Québec; Comeau, Y., L. Favreau, B. Lévesque et M. Mendell (2001). Emploi, économie sociale et développement local : les nouvelles filières, Sainte-Foy, Presses de l'Université du Québec.

2 Cornforth, C., A. Thomas, J. Lewis et R. Spear (1988). Developing Successful Worker Co-operatives, London, Sage Publications; Defourny, J. (1994). Développer l'entreprise sociale, Bruxelles, Fondation du Roi Baudouin; Bernoux, J.-F. (2005). Mettre en ouvre le développement social territorial, Paris, Dunod; Mondolfo, P. (2005). Conduire le développement social, Paris, Dunod.

3 Weil, M. (dir.) (2005). Handbook Of Community Practice, Thousand Oaks, Sage Publications; Bourque, D., Y. Comeau, L. Favreau et L. Fréchette (dir.) (2007). L'organisation communautaire. Fondements, approches et champs de pratiques, Québec, Presses de l’Université du Québec.

4 Définition inspirée de Lachapelle, R. (dir.) (2003). L'organisation communautaire en CLSC. Cadre de référence et pratiques, Presses de l'Université Laval.

5 La recherche a bénéficié de l'appui financier du Conseil de recherche en sciences humaines du Canada.

6 En 1977, le Regroupement des organisateurs communautaires du Québec (ROCQ) est fondé. En devenant le Collectif québécois de conscientisation en 1979, ses fondateurs et ses adhérents veulent refléter la mouvance et le modèle d'intervention qui les inspire, soit la conscientisation. Toutefois, l'association réunissant des intervenants sur la base du métier d'organisation communautaire est davantage le fait du Regroupement québécois des intervenants et intervenantes en action communautaire en CLSC et en centres de santé (RQIIAC), créé en 1988. 
7 Mercier, C. (2000). «L'organisation communautaire et le travail social », dans J.-P. Deslauriers et Y. Hurtubise dir.), Introduction au travail social, Québec, Les Presses de l’Université Laval.

8 Du côté associatif sont rejoints les associations de défense des droits, les organismes d'aide aux démunis (sauf l'aide alimentaire), les organismes d'aide humanitaire et de solidarité internationale, les centres communautaires, les centres de loisirs, les organismes environnementaux, les fonds locaux et régionaux de capital de risque, les médias communautaires et engagés, les groupes de ressources techniques, les maisons des jeunes, les regroupements régionaux d'organismes, etc.. Sont également contactés les Carrefours Jeunesse Emploi, les structures syndicales régionales, les Centres locaux de développement et les organismes de pastorale sociale.

9 Trois raisons motivent ce choix : 1) le réalisme, car étant donné qu'il existe plusieurs milliers d'organismes commu- nautaires, syndicaux et parapublics au Québec, la tâche d'identifier les OC apparaît titanesque; 2) ces régions présentent des profils urbain et rural qui permettent théoriquement de considérer, sur ce plan, la variété d'organismes; 3) ces territoires sont bien connus par les membres de l'équipe et sont situés à proximité de leur université respective.

10 La définition de l'organisation communautaire qui était soumise est : «1) je fais une intervention salariée avec un groupe de personnes, une ou plusieurs associations, ou une collectivité de type territorial, identitaire ou d'intérêt; 2) mon intervention salariée est pratiquée selon un processus par lequel ce groupe, cette association ou cette collectivité identifie des problèmes, mobilise des ressources et développe une action collective pour y répondre (par exemple, sensibilisation, éducation, défense des droits, action directe, économie sociale, etc.); 3 ) mon intervention salariée est orientée vers la solidarité et la participation sociale au moyen de pratiques démocratiques $»$. 


\section{Publicité}

CEE 